\title{
Ad-shWnt2b Vector Therapy Demonstrates Antitumor Activity in Orthotopic Intrapleural Models as Monitored with the In Vitro Imaging System (IVIS)
}

\author{
MASASHI KOBAYASHI ${ }^{1}$, CHENG-LONG HUANG ${ }^{2}$, MAKOTO SONOBE ${ }^{2}$, \\ RYUTARO KIKUCHI ${ }^{2}$ and HIROSHI DATE ${ }^{2}$ \\ ${ }^{1}$ Department of Thoracic Surgery, Tokyo Medical and Dental University, Tokyo, Japan; \\ ${ }^{2}$ Department of Thoracic Surgery, Kyoto University, Kyoto, Japan
}

\begin{abstract}
Aim: We investigated whether Wnt2B-inhibiting therapy has effective antitumor activity against Wnt $2 B$ overexpressing cells in an orthotopic intrapleural model by monitoring with the in vitro imaging system (IVIS). Materials and Methods: Mice for the orthotopic intrapleural model were randomized into 3 groups (control, Ad-shWnt $2 B$ and Ad-scramble; 8 mice per group). The respective vector was injected into the intrapleural cavity of mice in the AdshWnt2B and Ad-scramble groups. After 6 weeks of vector treatment, all mice were monitored with the IVIS. Additionally, their body weight was measured until all mice died from the tumor or were sacrificed. Results: A549-Lucpositive cells showed cytotoxicity following exposure to the Ad-shWnt $2 B$ vector. The percentage of viable cells was significantly lower in A549-Luc cells treated with AdshWnt $2 B$ than with Ad-scramble ( $p<0.01$ versus control or Ad-scramble, respectively).
\end{abstract}

In vitro detection of A549-Luc cells revealed that photon counts were highly correlated with the number of cells in the test wells $\left(r^{2}=0.98\right)$. IVIS monitoring of the orthotopic intrapleural model during treatment showed a rapid increase in the photon-flux count after day 14 in the control and Adscramble-treated groups. In contrast, the photon intensity of the Ad-shWnt2B-treated group was significantly lower than that of the Ad-scramble-treated and control groups after day 14 ( $p<0.01$ versus control or Ad-scramble, respectively). Marked weight loss of $15 \%$ was observed in both the control

Correspondence to: Masashi Kobayashi, Department of Thoracic Surgery, Tokyo Medical and Dental University, Bunkyo-ku, Tokyo, Japan. Tel: +81 358034072, Fax: +81 358030375, e-mail: mkobthsr@tmd.ac.jp

Key Words: Wnt2b, orthotopic model, IVIS, adenoviral vector, A549, luciferase. and Ad-scramble groups after day 77 compared with the AdshWnt2B group. In addition, the overall survival was significantly lower in the control and Ad-scramble groups than in the Ad-Wnt2B group ( $p<0.01$ versus Ad-shWnt2B, respectively). Treatment with the Ad-shWnt2B vector did not elicit any side-effects during this study. Conclusion: AdshWnt2B therapy had effective antitumor activity against the Wnt2B-overexpressing cell line in the orthotopic intrapleural model as monitored with the IVIS.

The Wnt family is involved in the regulation of a wide variety of normal and pathological functions in tumor tissues $(1,2)$. Our previous reports have described several Wnt family members as significant oncogenes in lung cancer and malignant pleural mesothelioma (3-5).

In particular, Wnt2B is known to stimulate the canonical Wnt/B-catenin pathway $(6,7)$ the activation of which leads to the transcription of Wnt target genes, including c-Myc (8) and the genes coding for survivin $(8,9)$ and vascular endothelial growth factor A (10). Therefore, it is possible that Wnt2B overexpression may affect the tumor biology and progression through the induction of these tumor-associated Wnt targets (3).

The adenoviral vectors from human adenovirus serotype 5 with lack of the $E 1$ and $E 3$ genes are widely available for expression studies of transgenes using the efficient small interfering RNA (siRNA) into the cells (12-16). Stable siRNA molecules can be produced via short hairpin RNA (shRNA) expressed under the control of the RNA polymerase IIIdependent promoter $(17,18)$. Consequently, we prepared an adenoviral vector expressing the short hairpin RNA (shRNA) against targets of Wnt2B (19). In the present study, we investigated whether Wnt2B-inhibiting therapy could have effective antitumor activity against Wnt2B-overexpressing cancer cells in the orthotopic intrapleural model as monitored by the in vivo imaging system (IVIS), which has demonstrated positive correlation between the number of cancer cells and strength of photons. The photon intensity 


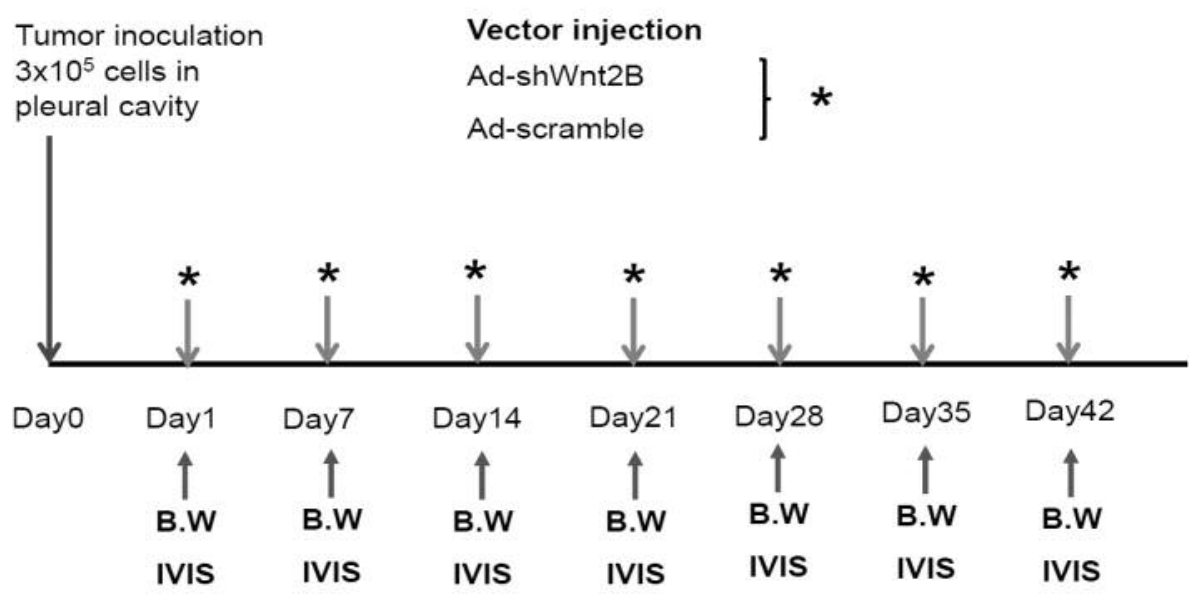

Figure 1. Treatment protocol for orthotopic intrapleural implantation models with Wnt2B-overexpressing tumor cells. $3 \times 10^{5}$ A549-Luc cells in $100 \mathrm{ml} \mathrm{FBS-free} \mathrm{medium} \mathrm{were} \mathrm{injected} \mathrm{into} \mathrm{the} \mathrm{pleural} \mathrm{cavity} \mathrm{of} \mathrm{six-week-old} \mathrm{BALB} \mathrm{mice.} \mathrm{The} \mathrm{same} \mathrm{technique} \mathrm{was} \mathrm{used} \mathrm{for} \mathrm{the} \mathrm{intrapleural}$ injections of Ad-shWnt $2 B$ or Ad-scramble for 6 weeks. Mice were randomized into Ad-shWnt $2 B$ or Ad-scramble groups and a control group (8 mice per group). The total study period was 6 weeks.

from the tumor is proportional to the number of light-emitting cells and the signal can be measured to monitor tumor growth and development $(20,21)$.

\section{Materials and Methods}

Cell line and culture conditions. The A549-luciferase cell line (A549-Luc; human lung cancer cell line, adenocarcinoma) was a gift from Dr. Maekawa (Department of Transfusion Medicine and Cell Therapy, Kyoto University, Japan). Cells were maintained in RPMI 1640 medium supplement with $10 \%$ fetal bovine serum (FBS) and grown in atmosphere containing $5 \%$ at $37^{\circ} \mathrm{C}$.

To evaluate how the number of cells affects the photon counts, A549-Luc cells were diluted to $1.0 \times 10^{5}, 5.0 \times 10^{4}, 2.5 \times 10^{4}$ and $1 \times 10^{4}$ cells per well. A blank of medium with no cells was included. The cells were measured by the IVIS $4 \mathrm{~min}$ after $150 \mu \mathrm{g} / \mathrm{ml}$ of Dluciferin had been added to each well.

Preparation of $\operatorname{shWnt} 2 B$ adenoviral vector. We prepared an adenoviral vector expressing the short hairpin RNA (shRNA) against the target of Wnt2B (Ad-shWnt2B) (19). The Ad-shWnt2B vector inhibited the growth of Wnt2B-overexpressing tumors and increased their apoptosis efficiently. This proved that Ad-shWnt2B has a strong antitumor effect against Wnt2B-overexpressing tumors. The Ad-shWnt2B vector, which was injected into the mouse intrapleural cavity at $2 \times 10^{9}$ plaque-forming units (PFUs) $/ \mathrm{ml}$, was kept frozen at $-80^{\circ} \mathrm{C}$ until use. We also prepared a control adenoviral vector expressing shRNA against the scrambled sequence of Wnt2B (Ad-scramble). Production of these vectors was realized in collaboration with the Kagawa University.

MTS assay. Cell viability was determined by carrying out the MTS (3-(4,5-dimethylthiazol-2-yl)-5-(3-carboxymethoxyphenyl)-2-(4sulfophenyl)-2H-tetrazolium) assay using a cell counting kit (Dojindo, Kumamoto, Japan).
To assess the cytotoxic effect of the virus, A549-Luc cells were seeded into 96-well plates at $3 \times 10^{4}$ cells per well and after $48 \mathrm{~h}$ were infected with the Ad-shWnt2B vector at $\times 1, \times 10, \times 20$ or with the Adscramble vector. At indicated times post-infection, $10 \mu \mathrm{l}$ of MTS solution was added to each well and the cells were incubated for $2 \mathrm{~h}$ at $37^{\circ} \mathrm{C}$. Cell viability was determined by measuring the absorbance at $490 \mathrm{~nm}$ using a microplate reader (iMark; BioRad Lab, Berkeley, CA, USA). Control groups at each time point were set as $100 \%$ viability.

Orthotopic intrapleural models with Wnt2B-overexpressing tumor cells. Six-week-old male BALB/c/mu/mu mice were purchased from Clea Japan Inc. (Tokyo, Japan). All animal experiments were approved by the Committee of Ethics on Animal Experiments in Kyoto University and carried out according to the Guidelines for Animal Welfare Committee of Kyoto University.

On day 0 , A549-Luc cells $\left(3 \times 10^{5}\right.$ cells in $100 \mu$ of FBS-free medium) were injected with a 27 -gauge needle into the left pleural cavity of BALB/c mice that had been placed under general anesthesia with isoflurane. The IVIS was leveraged to monitor whether the orthotopic intrapleural model worked. Established orthotopic intrapleural models were then randomized into the control, AdshWnt2B vector and Ad-scramble vector groups ( 8 mice per group) at day 1 . The same technique was used for both the Ad-shWnt $2 \mathrm{~B}$ and Ad-scramble intrapleural injections for 6 weeks starting on day 1 .

All orthotopic intrapleural implantation models were monitored with the IVIS where optical imaging was carried out on days 1, 7, 14, 21, 28, 35 and 42 (Figure 1). After 35 days, treatment and monitoring with the IVIS were ceased in all mice but the measurement of body weight was continued until all mice had died from the tumor or were sacrificed.

Measure of bioluminescence imaging with the IVIS. Mice were anesthetized by isoflurane inhalation after an initial anesthesia with a mixture of $2 \%$ isoflurane and oxygen $(2 \mathrm{l} / \mathrm{min})$. In an induction chamber, the mice were injected intraperitoneally with $100 \mu \mathrm{l}$ of the D-luciferin solution (150 mg/kg body weight). 


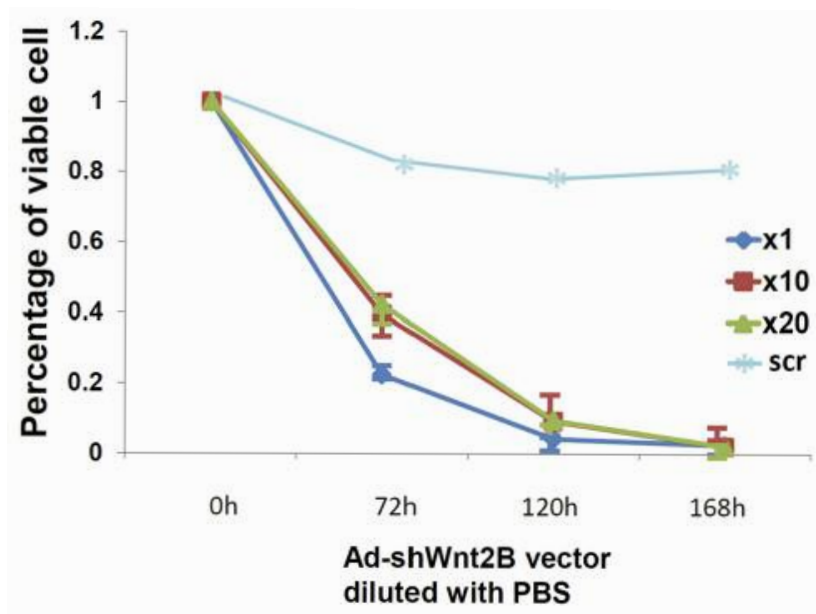

Figure 2. Ad-shWnt $2 B$ showed dose- and time- dependent cytotoxicity against A549-Luc cell by MTS assay. Transduction with Ad-shWnt2B strongly reduced the percentage of viable cells in A549-Luc cells in a dose- and time-dependent manner. Ad-shWnt $2 B$ vector was properly diluted from the titer of $2 \times 10^{9}$ PFUs (see Materials and Methods).

At 10 min after injection, the animals were anesthetized in an induction chamber and placed in the IVIS chamber in a standardized way. Images based on the luciferin activity were acquired for 1-60 s with the mice maintained under general anesthesia via a nasal cone. Each treatment group of 5 mice was placed in the IVIS chamber mounted to the charge-coupled device camera cooled to $-120^{\circ} \mathrm{C}$, with a field of view set at $25 \mathrm{~cm}$ above the sample shelf.

Bioluminescence, transmitted from tumor cells in the left pleural cavity, was observed by measuring the mice in the left lateral position. Background photon images and bioluminescence color images were superimposed using Living Image V. 2.11 software overlay (Caliper Life Sciences, Hopkinton, MA, USA) and IGOR image analysis software (V. 4.02 A; WaveMetrics, Lake Oswego, OR, USA). A region of interest was manually selected over the signal intensity. Values were expressed in photons per second per squared-centimeter $\left(\mathrm{p} \cdot \mathrm{s}^{-1} \cdot \mathrm{cm}^{-2}\right)$ and represent the mean values from the left lateral position of each animal. All bioluminescence data were collected and analyzed using the IVIS.

Statistical analysis. The data are shown as the mean \pm standard error (SE). All statistical analyses, including the comparison of continuous data between groups, were performed using StatView V. 5.0 (SAS Institute Inc., Cary, NC, USA). The Student's $t$-test was applied to determine the number of photon counts. The post-treatment survival rate was analyzed by the Kaplan-Meier method, whereas the difference in survival rates was evaluated by the log-rank test. A $p$ value of less than 0.05 was considered to be statistically significant.

\section{Results}

MTS assay. The in vitro MTS assay showed dose- and timedependent cytotoxicity against A549-Luc cells. A549-Lucpositive cells showed cytotoxicity following exposure to the As-shWnt2B vector. The percentage of viable cells was
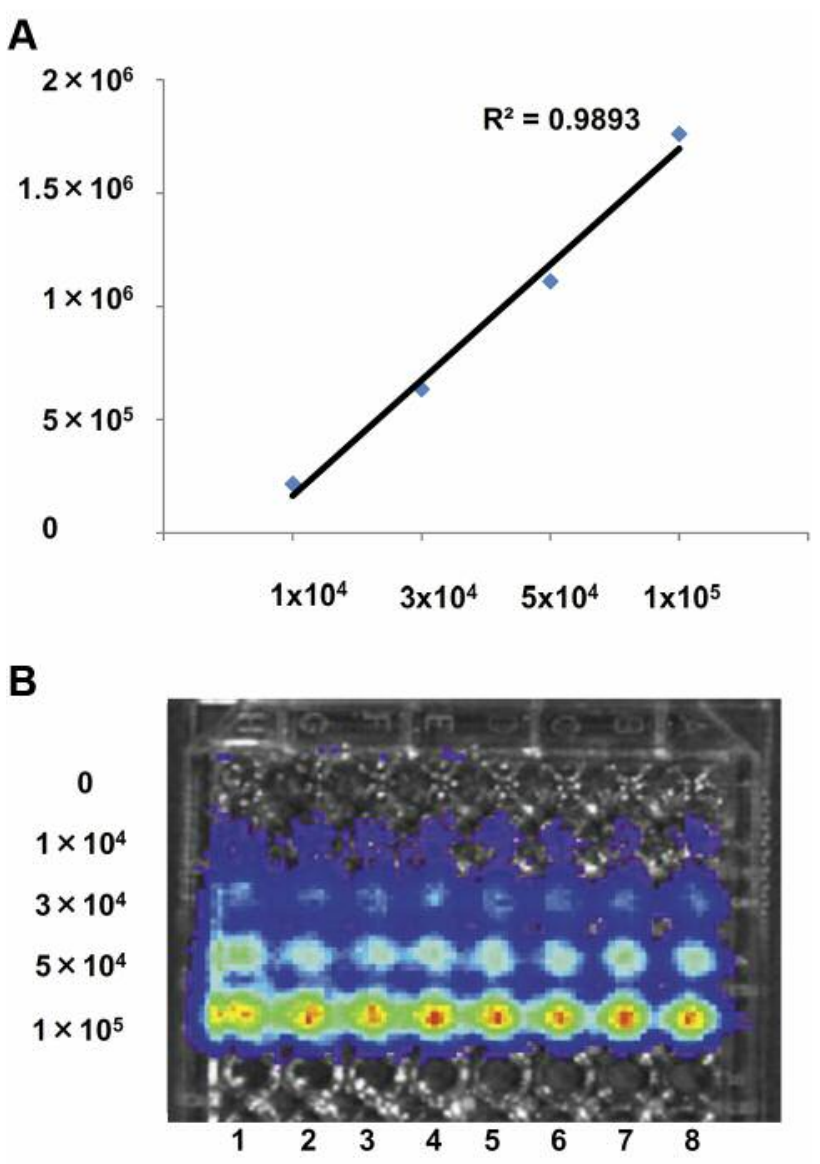

Figure 3. Correlation of tumor cell numbers and photon counts. A: Correlation curve: $R^{2}=0.9893$. B: Photon counts were highly correlated to the number of cells in each well $\left(r^{2}=0.98\right)$ as measured by IVIS $4 \mathrm{~min}$ after the injection of D-luciferin.

significantly lower in the A549-Luc cells treated with AdshWnt2B than with Ad-scramble $(p<0.01$ versus control and Ad-scramble, respectively) (Figure 2). The transmission of the Ad-shWnt2B vector strongly reduced the percentage of viable A549-Luc cells in dose- and time-dependent manners.

Tumor cells in relation to photon counts. In vitro detection of A549-Luc cells revealed the photon counts to be highly correlated to the number of cells in each well $\left(r^{2}=0.98\right)$ (Figure 3A) as measured by the IVIS at $4 \mathrm{~min}$ after the injection of D-luciferin. The IVIS determined an exponential increase in the bioluminescence activity in relation to the cell number (Figure3B).

IVIS monitoring of the orthotopic intrapleural implantation model during treatment. Orthotopic intrapleural implantation models were prepared using nude mice. After treatment, the bioluminescence intensity in each sample was monitored using 


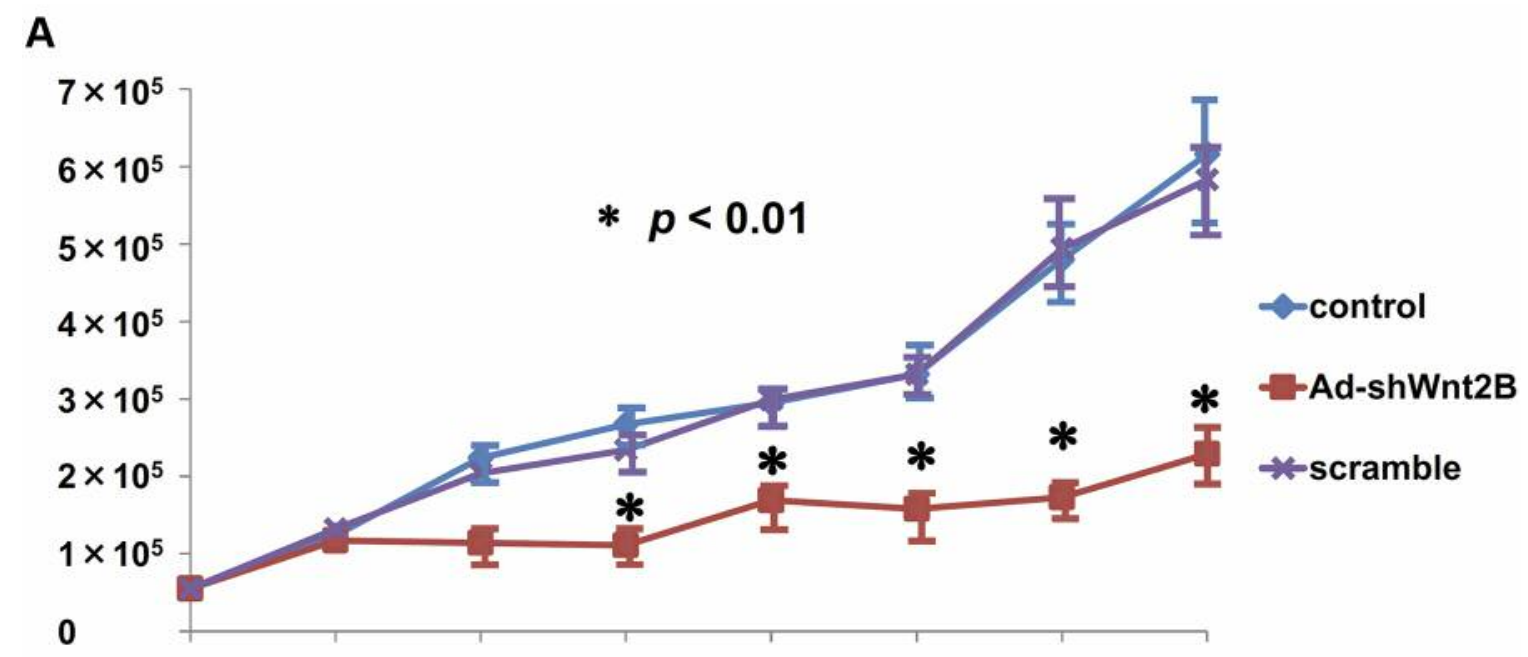

Day0 Day1 Day7 Day14 Day21 Day28 Day35 Day42

B

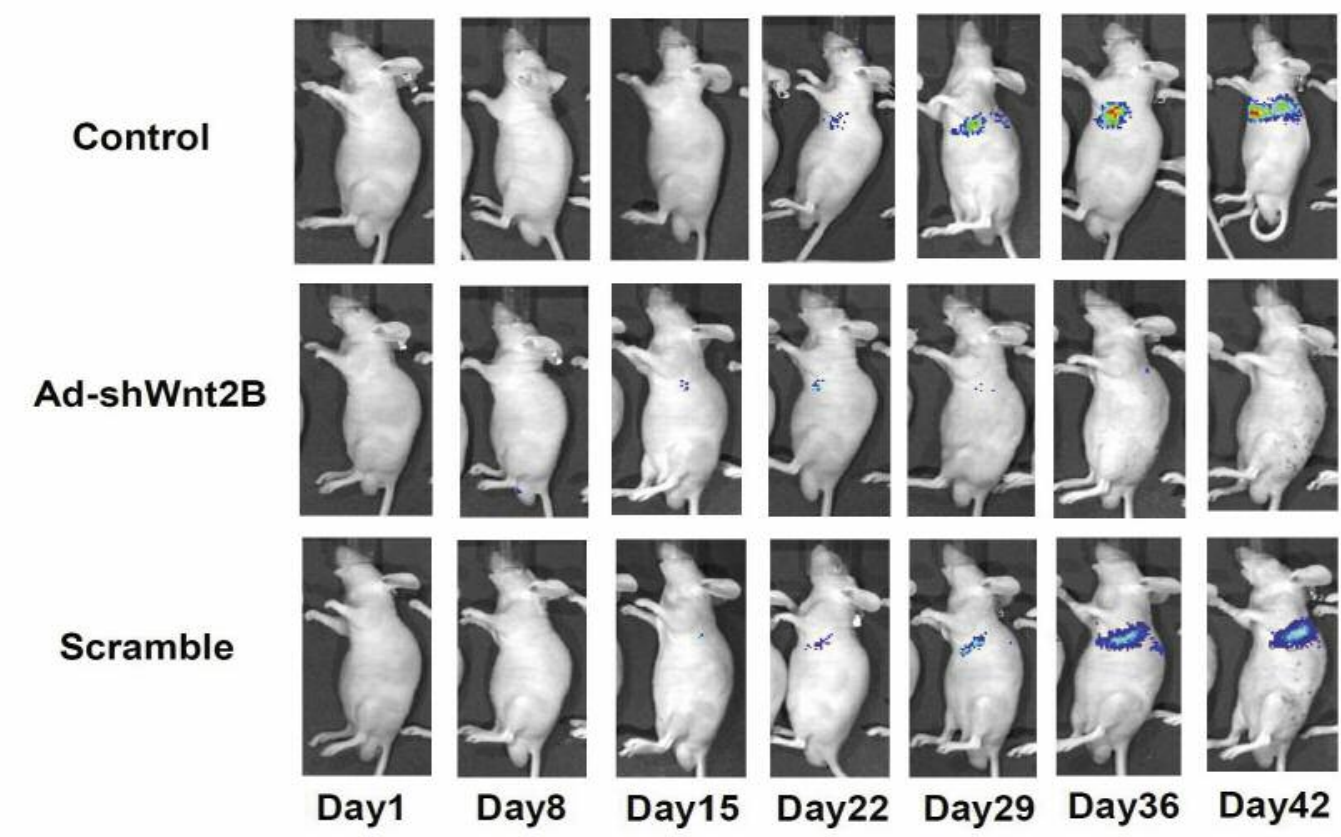

Figure 4. Photon intensity of A549-Luc cells in orthotopic models monitored with this IVIS system during vector treatment. A: The photon intensity of Ad-Wnt2B-treated groups was significantly lower than that of Ad-scramble-treated or control groups. B: Photon intensity of A549-Luc cells in orthotopic models monitored with the IVIS system.

the IVIS. The monitoring showed a disinhibitory increase in the photon-flux count after day 14 in the control and Adscramble-treated groups, whereas that of the Ad-shWnt2Btreated group was significantly lower $(p<0.01$ versus control and Ad-scramble, respectively) (Figure 4A and B).

Upon evaluation of the IVIS images, a serial increase of photon intensity was clearly observed in the left thoracic cavity in both the control and Ad-scramble groups but not in the Ad-shWnt2B group, which proved the therapeutic efficacy of Ad-shWnt2B.

Thus, treatment with the Ad-shWnt2B vector was demonstrated to have effective antitumor activity against the Wnt2B-overexpressing cells in the orthotopic intrapleural model; no side-effects were observed during this experiment. 


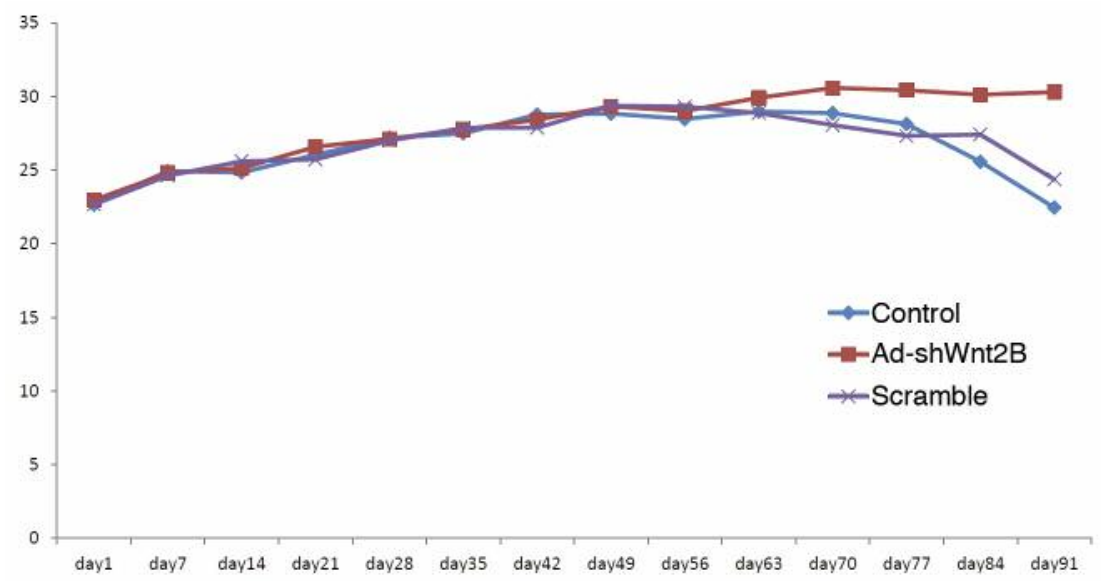

Figure 5. Body weight change during treatment. There was no difference in weight change between Ad-shWnt2B-treated groups and control or scramble groups.

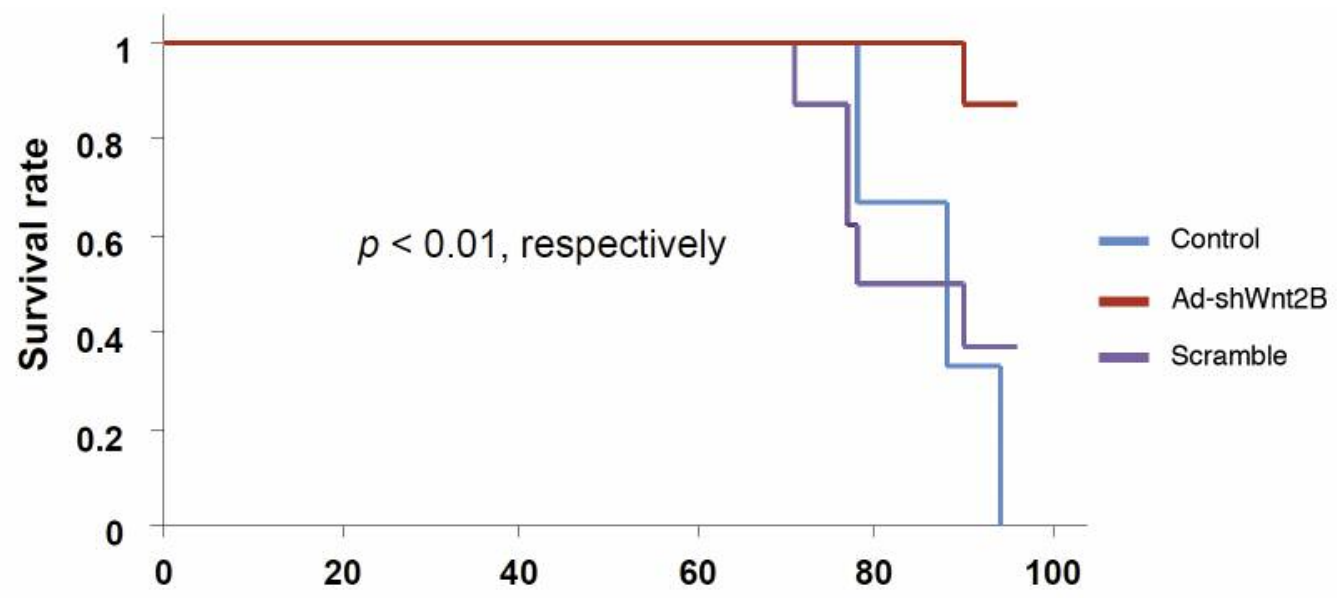

Figure 6. Survival of mice in relation to treatment status. The control and Ad-scramble-treated mice had a significantly lower survival rate ( $p<0.01$ versus $A d$-shWnt $2 B$, respectively) than mice treated with $A d-s h W n t 2 B$.

Body weight change during treatment. There was a marked weight loss of $15 \%$ in the control and Ad-scramble groups, compared to the Ad-shWnt2B group, after day 77 (Figure 5). Apart from the documented immense weight loss, an increase of mortality was also indicated in these 2 groups. In the Ad-shWnt2B treatment group, however, a steady body weight was maintained until all mice were sacrificed at the study's completion.

Survival of mice in relation to treatment status. The control and Ad-scramble-treated mice had a significantly lower survival rate ( $p<0.01$ versus Ad-shWnt2B, respectively) than the mice treated with Ad-shWnt2B (Figure 6). The photonflux count from the orthotopic intrapleural models was detected as early as day 7 post-inoculation, while tumor growth in the intrapleural cavity continued until all mice had died from the tumor or were sacrificed. Furthermore, a correlation between the increase of photon intensity in luciferase and the lower survival rate was observed during the experimental period.

\section{Discussion}

Several techniques, including surgical implantation or injection of cancer cell suspension, for obtaining an accurate orthotopic model have been reported. In this study, we constructed a pleural lesions model by percutaneous intrathoracic injection of cell suspension. Hoffman et al. 
have reported a patient-derived orthotopic xenograft (PDOX) model obtained by surgical orthotopic implantation of various cancer cells; however, a high-quality model could not be achieved (22-24).

Treatment processes that can be visualized permit to obtain important information about the biological activity in a study. Such monitoring systems are useful for directly evaluating the in vivo therapeutic effects of different drugs and treatment processes in animals. Biological reactions and therapeutic effects in an in vivo model have been reported in various carcinomas by using non-invasive visualization methods (25-31)

We chose to study the Wnt pathway since its regulation is a prevalent theme in cancer biology. Among various molecules, the Wnt gene family encodes multifunctional signaling glycoproteins that are involved in the regulation of a wide variety of normal and pathological processes, including embryogenesis and tumorigenesis (1, 2). Furthermore, many clinical studies have reported that overexpression of Wnt members is associated with various tumorigenic processes, such as proliferation, angiogenesis and patient survival (32-34). We, therefore, perceived that the mechanism of Wnt2B is important in carcinomas.

We previously demonstrated that intratumoral expression of Wnt2B was high in many malignant tumors, including malignant pleural mesothelioma and lung cancer $(3,19)$ and also involved in tumorigenesis and disease prognosis.

We hypothesized that inhibition of the Wnt2B signaling pathway, via the decreased expression of survivin and c-Myc, would result in interruption of tumor proliferation and induction of apoptosis. This hypothesis was proven accurate when the repression of survivin and $c-M y c$ by use of the Ad-shWnt2B vector was shown to induce tumor apoptosis in Wnt2Boverexpressing cells, including the A549 cell line (19).

Thus, we evaluated the effectiveness of Ad-shWnt2b intrapleural therapy against Wnt2B-overexpressing cancer cells in an orthotopic intrapleural model, monitored by the IVIS. Our in vivo model represents a "patient-like" form of malignant pleural effusion or malignant pleural mesothelioma.

In the present study, we evaluated the shWnt2B therapy by monitoring the immediate optical effects by using the IVIS. The first 2 weeks of treatment with Ad-shWnt2B resulted in effective repression of the luminescence intensity, compared to that of the Ad-scramble and control groups. Moreover, the Ad-shWnt2B-treated group maintained lower photon intensity than that of the non-treated groups after day 14. Survival prolongation and weight maintenance are the additional parameters for evaluating therapeutic effects. Our models showed that the Ad-shWnt2B-treated group acquired a high survival rate and maintained body weight, unlike the non-treated groups. Furthermore, the photon intensity in the Ad-shWnt2B group did not increase after cessation of treatment. These findings indicate that the Wnt2B-inhibiting therapy has an effective antitumor activity and that IVIS is an efficient monitoring technology, which provides accurate and fast therapeutic information.

Interestingly, luminescence measurement systems using molecules other than luciferase, such as green fluorescent protein (GFP), are available. Previous studies have reported that fluorescent protein imaging has significant advantages over luciferase imaging (29-31). Fluorescent protein imaging with GFP has led to ideas for potential drug development. In addition, this method was used to visualize single cancer cell level and it was possible to acquire patterns of tumor metastasis with the nuclear and cytoplasmic dynamics in living animals. Therefore, we expect that fluorescent protein imaging with GFP can accelerate the development of anticancer therapy.

In conclusion, the orthotopic intrapleural model was stably maintained throughout this study. Ad-shWnt2B showed effective antitumor activity in this in vivo model with Wnt2B-overexpressing A549-Luc cells, as monitored by the IVIS. Moreover, mice treated with Ad-shWnt2B showed no adverse effects during this study. Further studies on the combination therapy of Ad-shWnt2B and chemotherapy or treatment with a cationic vector (35-37) in the orthotopic intrapleural model would be useful to help develop this vector into an effective antitumor tool.

\section{References}

1 Dale TC: Signal transduction by the Wnt family of ligands. Biochem J 329: 209-223, 1998.

2 You Z, Saims D, Chen S, Guttridge DC, Guan KL, MacDougald OA, Brown AM, Evan G, Kitajewski J and Wang CY: Wnt signaling promotes oncogenic transformation by inhibiting cMyc-induced apoptosis. J Cell Biol 157: 429-440, 2002.

3 Kobayashi M, Huang C, Sonobe M, Kikuchi R, Ishikawa M, Kitamura J, Miyahara R, Menju T, Iwakiri S, Itoi K, Yasumizu $\mathrm{R}$ and Date $\mathrm{H}$ : Intratumoral Wnt2B expression affects tumor proliferation and survival in malignant pleuralmesothelioma patients. Exp Ther Med 3(6): 952-958, 2012.

4 Huang C, Liu D, Masuya D, Kameyama K, Nakashima T, Yokomise $\mathrm{H}$, Ueno $\mathrm{M}$ and Miyake $\mathrm{M}$ : MRP-1/CD9 gene transduction downregulates Wnt signal pathways. Oncogene 23: 7475-7483, 2004.

5 Huang C, Liu D, Ishikawa S, Nakashima T, Nakashima N, Yokomise H, Kadota $\mathrm{K}$ and Ueno M: Wnt1 overexpression promotes tumour progression in non-small cell lung cancer. Eur J Cancer 44: 2680-2688, 2008.

6 Katoh M, Kirikoshi H, Terasaki H and Shiokawa K: WNT2B2 mRNA, up-regulated in primary gastric cancer, is a positive regulator of the WNT-beta-catenin-TCF signaling pathway. Biochem Biophys Res Commun 289: 1093-1098, 2001.

7 Katoh M: WNT2B: comparative integromics and clinical applications. Int J Mol Med 16: 1103-1108, 2005.

8 He TC, Sparks AB, Rago C, Hermeking H, Zawel L, da Costa LT, Morin PJ, Vogelstein B and Kinzler KW: Identification of cMYC as a target of the APC pathway. Science 281: 1509-1512, 1998. 
9 Kim PJ, Plescia J, Clevers H, Fearon ER and Altieri DC: Survivin and molecular pathogenesis of colorectal cancer. Lancet 362: 205-209, 2003.

$10 \mathrm{Ma} \mathrm{H}$, Nguyen C, Lee KS and Kahn M: Differential roles for the coactivators $\mathrm{CBP}$ and $\mathrm{p} 300$ on $\mathrm{TCF} / \beta$-catenin-mediated survivin gene expression. Oncogene 24: 3619-3631, 2005.

11 Zhang X, Gaspard JP and Chung DC: Regulation of vascular endothelial growth factor by the Wnt and K-ras pathways in colonic neoplasia. Cancer Res 61: 6050-6054, 2001.

12 Anderson WF: Human gene therapy. Nature 392: 25-30, 1998.

13 Vile R: Cancer gene therapy - new approaches to tumor cell killing. J Gene Med 2: 141-143, 2000.

14 Hannon GJ: RNA interference. Nature 418: 244-251, 2002.

15 Scherer L and Rossi JJ: RNAi applications in mammalian cells. Biotechniques 36: 557-561, 2004.

16 Huang C, Li M, Chen C and Yao Q: Small interfering RNA therapy in cancer: mechanism, potential targets, and clinical applications. Expert Opin Ther Targets 12: 637-645, 2008.

17 Brummelkamp TR, Bernards R and Agami R: A system for stable expression of short interfering RNAs in mammalian cells. Science 296: 550-553, 2002.

18 Rao DD, Vorhies JS, Senzer N and Nemunaitis J: SiRNA vs. shRNA: similarities and differences. Adv Drug Deliv Rev 61: 746-759, 2009.

19 Lu D, Kadota K, Ueno M, Nakashima N, Yokomise H and Huang CL: Adenoviral vector expressing short hairpin RNA targeting Wnt2B has an effective antitumor activity against Wnt2B2overexpressing tumors. Eur J Cancer 48: 1208-1218, 2012.

20 Rice BW, Cable MD and Nelson MB: in vivo imaging of lightemitting probes. J Biomed Opt 6: 432-440, 2001.

21 Yanagihara K, Takigahira M, Takeshita F, Komatsu T, Nishio K, Hasegawa $\mathrm{F}$ and Ochiya T. A photon counting technique for quantitatively evaluating progression of peritoneal tumor dissemination. Cancer Res 66: 7532-7539, 2006.

22 Hoffman RM: Patient-derived orthotopic xenografts: better mimic of metastasis than subcutaneous xenografts. Nat Rev Cancer 15: 451-452, 2015.

23 Hoffman RM: Orthotopic metastatic mouse models for anticancer drug discovery and evaluation: A bridge to the clinic. Invest New Drugs 17: 343-360, 1999.

24 Wang, Xiaoen, X. Fu and Hoffman RM: A new patient-like metastatic model of human lung cancer constructed orthotopically with intact tissue via thoracotomy in immunodeficient mice. Int J Cancer 51: 992-995, 1992.

25 Matsumoto S, Tanaka F, Sato K, Kimura S, Maekawa T, Hasegawa $\mathrm{S}$ and Wada $\mathrm{H}$ : Monitoring with a non-invasive bioluminescent in vivo imaging system of pleural metastasis of lung carcinoma. Lung Cancer 66: 75-79, 2009.
26 Kadonosono T, Kuchimaru T, Yamada S, Takahashi Y, Murakami A, Tani $\mathrm{T}$, Watanabe $\mathrm{H}$, Tanaka $\mathrm{T}$, Hirota $\mathrm{K}$, Inoue $\mathrm{M}$, Tsukamoto T, Toyoda T, Urano K,Machida K, Eto T, Ogura T, Tsutsumi H, Ito M, Hiraoka M, Kondoh G and Kizaka-Kondoh $\mathrm{S}$ : Detection of the onset of ischemia and carcinogenesis by hypoxia-inducible transcription factor-based in vivo bioluminescence imaging. PLoS One 6(11): e26640, 2011.

27 Luker KE, Mihalko LA, Schmidt BT, Lewin SA, Ray P, Shcherbo D, Chudakov DM and Luker GD: In vivo imaging of ligand receptor binding with Gaussia luciferase complementation. Nat Med 18: 172-177, 2011.

28 Martínez-Corral I, Olmeda D, Diéguez-Hurtado R, Tammela T, Alitalo K and Ortega S: In vivo imaging of lymphatic vessels in development, wound healing, inflammation, and tumor metastasis. Proc Natl Acad Sci USA 109: 6223-6228, 2012.

29 Hoffman RM: The multiple uses of fluorescent proteins to visualize cancer in vivo. Nat Rev Cancer 5: 796-806, 2005.

30 Hoffman RM and Meng Y: Subcellular imaging in the live mouse. Nature Protocols 1: 775-782, 2006.

31 Hoffman RM and Meng Y: Whole-body imaging with fluorescent proteins. Nat Protoc 1: 1429-1438, 2006.

32 Huang C, Liu D, Nakano J, Ishikawa S, Kontani K, Yokomise $\mathrm{H}$ and Ueno M: Wnt5a expression is associated with the tumor proliferation and the stromal vascular endothelial growth factorA expression in non-small cell lung cancer. J Clin Oncol 23: 8765-8773, 2005.

33 Chen G, Shukeir N, Potti A, Sircar K, Aprikian A, Goltzman D and Rabbani SA: Up-regulation of Wnt-1 and $\beta$-catenin production in patients with advanced metastatic prostate carcinoma: potential pathogenetic and prognostic implications. Cancer 101: 1345-1356, 2004.

34 Zhang WM, Lo Muzio L, Rubini C and Yan G: Effect of WNT1 on $\beta$-catenin expression and its relation to $\mathrm{Ki}-67$ and tumor differentiation in oral squamous cell carcinoma. Oncol Rep 13: 1095-1099, 2005.

35 Niidome T and Huang L: Gene therapy progress and prospects: Nonviral vectors. Gene Ther 9: 1647-1652, 2002.

36 Ozpolat B, Sood AK and Lopez-Berestein G: Liposomal siRNA nanocarriers for cancer therapy. Adv Drug Del Rev 66: 110-116, 2014.

37 Jin L, Zeng X, Liu M, Deng Y and He N: Current progress in gene delivery technology based on chemical methods and nanocarriers. Theranostics 4: 240-255, 2014.

Received July 18, 2016

Revised August 26, 2016

Accepted August 30, 2016 